\title{
Efectos de la cocaína sobre los niveles de $\gamma$-aminobutirato, glutamato y aspartato en el núcleo accumbens e hipocampo de rata
}

\author{
Rafael A. Ulloque \\ Facultad de Medicina, Universidad de Cartagena, Cartagena de Indias, Colombia.
}

Se estudiaron los efectos agudos y crónicos de la cocaína sobre los niveles de $\gamma$-aminobutirato (GABA), glutamato y aspartato en el núcleo accumbens e hipocampo de ratas, para lo cual se administró cocaína en forma aguda y crónica (intraperitoneal) a grupos de ratas. El primer grupo recibió $30 \mathrm{mg} / \mathrm{kg}$; la administración crónica se inició con $30 \mathrm{mg} / \mathrm{kg}$ y se incrementaron 5 $\mathrm{mg} / \mathrm{kg}$ cada cuatro días durante 20 días. Los grupos controles recibieron el respectivo volumen de solución salina, por la misma vía y por el tiempo respectivo. A los 30 minutos de administrada la cocaína en forma aguda y 24 horas después de la última dosis crónica, los animales fueron decapitados, los cerebros separados rápidamente del cráneo y colocados en solución salina normal a $0^{\circ} \mathrm{C}$ durante 10 minutos. Posteriormente, se tomaron muestras del núcleo accumbens y del hipocampo. Se procedió igual con los grupos controles.

Los niveles de GABA, glutamato y aspartato se determinaron utilizando técnicas enzimáticas y medidas espectrofotométricas.

Los resultados muestran que la administración aguda o crónica de cocaína incrementa significativamente el glutamato y el aspartato tanto en el núcleo accumbens como en el hipocampo. Además, se observó un descenso significativo del GABA con la administración crónica.

Estos resultados experimentales sustentan la hipótesis de que la administración de cocaína produce efectos excitadores, posiblemente, a través del incremento de las actividades glutaaspartatérgicas o a través de una disminución de actividad GABAérgica en el núcleo accumbens y en el hipocampo.

Palabras clave: cocaína y neurotransmisores aminoácidos, cocaína, núcleo accumbens, hipocampo.

Effects of cocaine on $\gamma$-aminobutyrate, glutamate and aspartate levels in rat nucleus accumbens and hippocampus

The acute and chronic effects of cocaine on $\gamma$-aminobutyrate (GABA), aspartate and glutamate levels both in nucleus accumbens and hippocampus were investigated. Doses of cocaine were administered intraperitoneally to groups of rats at levels to produce acute and chronic physiological responses. The acute treatment consisted of a dose of $30 \mathrm{mg} / \mathrm{kg}$. The chronic treatment was initiated with a dose of $30 \mathrm{mg} / \mathrm{kg}$ and then increased $5 \mathrm{mg} / \mathrm{kg}$ at 4-day intervals up to 20 days. The control groups were treated with the corresponding volume of saline solution with the same route of administration and at the same times. Thirty minutes after the acute dose and 24 hours after the last dose in the chronic group, the animals were decapitated and the brain quickly removed and placed in saline solution at $0{ }^{\circ} \mathrm{C}$ for 10 minutes. Control groups were treated identically.

GABA, aspartate and glutamate levels were measured by enzymatic assays with the aid of a spectrophotometer. A significant increase of aspartate and glutamate was recorded in the nucleus accumbens and hippocampus after acute or chronic administration of cocaine. The chronic treatment induced significant decrease of GABA levels in both areas. These data support the hypothesis that cocaine produces its excitatory effects possibly through an increase of gluta- 
aspartatergic activity and/or a decrease of GABAergic activity in the nucleus accumbens and hippocampus.

Key words: cocaine and amino acid neurotransmitters, cocaine, nucleus accumbens, hippocampus.

Existen sustancias que pueden ser drogas, fármacos o tóxicos capaces de inducir la dependencia y el abuso. Las drogas son sustancias sicoactivas cuya acción reforzante positiva es capaz de generar dependencia física, psíquica o ambas y que ocasionan en muchos casos un grave deterioro sicoorgánico y de la conducta social; esto puede ir acompañado o no de tolerancia y se caracteriza por modificaciones del comportamiento o por otras reacciones que comprenden siempre una compulsión a ingerirlas de un modo continuo o periódico con el fin de obtener sus efectos psíquicos y, a veces, para evitar el malestar de su privación (1).

Casi todas las culturas antiguas y contemporáneas han utilizado sustancias que alteran la conducta, el pensamiento y los sentimientos. Siempre existen en una sociedad individuos o grupos de individuos que tratan de innovar o contradecir las costumbres y tradiciones con relación a su uso. Existen registros antiguos del pueblo sumerio, del siglo II a.C., que relatan sobre los cultivos y el uso del opio con fines recreativos y medicinales. Se tiene noción también del uso del opio aún en los pueblos babilónicos y en el antiguo Egipto (2).

La tendencia del hombre a abusar de ciertas drogas y fármacos es compartida con algunos mamíferos inferiores utilizados en el laboratorio, que aprenden fácilmente a autoadministrarse sustancias como opiáceos, barbitúricos, alcohol y cocaína, entre otras. Se ha sugerido que esta conducta tiene un sustrato anatómico y neurobioquímico con la existencia de áreas cerebrales relacionadas. Hace más de tres décadas y media se propuso que un sistema monoaminérgico sería la base neurobioquímica de este circuito del placer (3).

\section{Correspondencia:}

Facultad de Medicina, Universidad de Cartagena, Cartagena Colombia.

Recibido: 18/04/01; aceptado: 06/07/01
A principios de los 60 se comenzó a publicar artículos que sostenían la idea de que, ciertamente, la actividad monoaminérgica juega un papel importante en el refuerzo del abuso de drogas. Básicamente, se trata de un sistema que conecta la parte ventral del cerebro medio con la parte ventral y anterior del encéfalo. Existen conexiones claves como son aquéllas que van de los tubérculos olfatorios, septum, núcleo accumbens al hipotálamo y a la región ventral tegmental. Además, existen vías ascendentes monoami-nérgicas hacia la parte media y anterior ventral del cerebro. Entre estas últimas, las más conocidas son las vías mesolímbica y mesocorticales, que son principalmente dopaminérgicas (4).

Una de las estructuras más citadas y estudiadas de este sistema de recompensa es el núcleo accumbens. Se piensa que esta estructura juega un papel clave en las acciones y efectos agudos y crónicos por las drogas de abuso. Se considera que la dopamina, otras monoaminas, neurotransmisores aminoácidos y neuropéptidos tienen gran actuación en las funciones de este centro de reforzamiento. Además, existen estructuras como el hipocampo, la corteza prefrontal, la corteza entorrinal, la amígdala cerebral y el globo pálido, las cuales también son importantes en el refuerzo de conductas y alteraciones neurobioquímicas por drogas que producen dependencia o adicción $(5,6)$.

Se ha informado extensamente que la cocaína actuaría principalmente en el sistema nervioso central (SNC), incrementando las concentraciones de dopamina y otras monoaminas en el espacio sináptico mediante un bloqueo de su recaptación en el pie terminal axónico, actuando sobre proteínas transportadoras específicas. Las propiedades estimulantes en animales de laboratorio (euforizantes, adictivas, cambios de la conducta) tratan de explicarse básicamente por la acción de la cocaína sobre los sistemas dopaminérgicos, acción especialmente importante sobre las vías meso-cortico-límbicas y nigro- 
estriadas, las principales vías dopaminérgicas en el SNC (3).

Sin embargo, otros sistemas podrían estar involucrados. Este trabajo es un aporte en la exploración de la posible participación de sistemas no dopaminérgicos, involucrados con la farmacodinamia de la cocaína. Aquí se informa de las alteraciones en los neurotransmisores: ácido $\gamma$-aminobutírico (GABA), glutamato y aspartato, por la administración de cocaína.

\section{Materiales y métodos}

Se organizaron tres grupos de 10 ratas machos Fisher 344 cada uno, obtenidas del bioterio del Instituto Nacional de Salud. Un grupo control se subdividió en dos con igual número de animales, y se le administró solución salina (intraperitoneal) aguda durante 20 días como tratamiento. Otro grupo de tratamiento agudo recibió clorhidrato de cocaína (de la casa Merck de Alemania, proporcionada por el Fondo Nacional de Estupefacientes) en dosis de $30 \mathrm{mg} / \mathrm{kg}$ en solución salina (intraperitoneal). Al tercer grupo se le administró clorhidrato de cocaína (por la misma vía) con dosis inicial de $30 \mathrm{mg} / \mathrm{kg}$ en solución salina con incremento de $5 \mathrm{mg} / \mathrm{kg}$ cada 4 días durante 20 días.

Pasados 30 minutos del tratamiento agudo y 24 horas después de la última dosis en los grupos crónicos, se sacrificaron los animales por decapitación. Los cerebros se removieron del cráneo en un tiempo promedio de 45 segundos, colocándolos inmediatamente en solución salina a $0^{\circ} \mathrm{C}$, durante 10 minutos. Luego de este período, se tomaron muestras del núcleo accumbens y del hipocampo.

\section{Ubicación y extracción del núcleo accumbens}

Se hicieron cortes coronales, $2 \mathrm{~mm}$ anterior al quiasma óptico (figura 1), de acuerdo con las coordenadas estereotáxicas establecidas por Paxinos y Watson (7). Los cortes de tejidos obtenidos se colocaron sobre un bloque de hielo con la ayuda de papel parafinado y mediante un micropunch (2,5 mm de diámetro) se extrajeron muestras del núcleo accumbens, cuyo peso osciló entre 25 y $30 \mathrm{mg}$ de tejido; luego, fueron homogenizadas en $1,5 \mathrm{ml}$ de alcohol etílico al $75 \%$ $(\mathrm{pH} 6,8)$ con la ayuda de ácido perclórico $(0,6 \mathrm{M})$

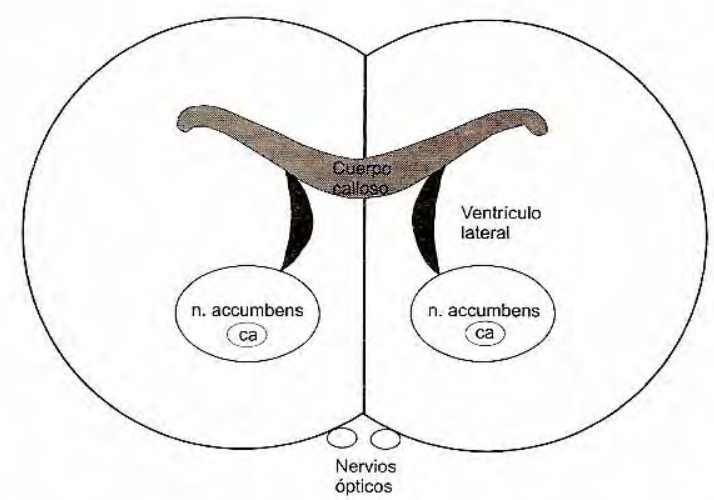

Figura 1. Diagrama del corte coronal de cerebro de rata (2 $\mathrm{mm}$ anterior al quiasma óptico) donde se muestra la ubicación y área tomada del núcleo accumbens.

ca: comisura anterior

a $0{ }^{\circ} \mathrm{C}$. Tras centrifugación a $10.000 \mathrm{rpm}\left(4{ }^{\circ} \mathrm{C}\right)$ durante 10 minutos, se extrajo el sobrenadante y se almacenó a $-40{ }^{\circ} \mathrm{C}$ hasta la realización de los ensayos (24-72 horas) para determinar los niveles de GABA, glutamato y aspartato.

\section{Ubicación y extracción del hipocampo}

Para la ubicación del hipocampo, se hicieron cortes coronales de $2 \mathrm{~mm}$ de espesor, $1 \mathrm{~mm}$ delante de la comisura pontocereberal (figura 2), identificándose muy bien el hipocampo por su forma semilunar. Estos se colocaron en hielo con la ayuda de papel parafinado y se tomaron porciones de tejidos de peso comprendido entre 55 y $60 \mathrm{mg}$ correspondientes a las zonas $\mathrm{CA}_{1}$, $\mathrm{CA}_{2}$ y $\mathrm{CA}_{3}$ del hipocampo (con la ayuda de un

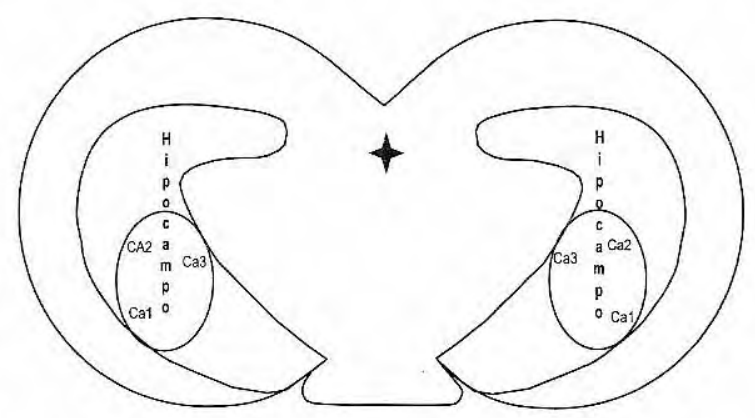

Figura 2. Diagrama del corte coronal de cerebro de rata (1 $\mathrm{mm}$ delante de la comisura pontocerebral) que muestra la extensión del hipocampo y la zona tomada, donde hay presencia de las células CA1, CA2 yCA3. 
micropunch, $4 \mathrm{~mm}$ de diámetro). La muestra se procesó igual como se hizo con el núcleo accumbens, hasta la congelación del sobrenadante (7).

Para la determinación de GABA en las muestras, se pipetearon en una microcelda de $1,5 \mathrm{ml}$ los siguientes reactivos: 948 uL de solución tampón de pirofosfato de sodio $(0,1 \mathrm{M} ; \mathrm{pH}=8,6) ; 55 \mathrm{uL}$ de $\mathrm{NADP}^{+}(4 \mathrm{mM}, \mathrm{pH}=7,0) ; 40 \mu \mathrm{L}$ de 2-mercaptoetanol $(0,06 \mathrm{M}), 40 \mu \mathrm{L}$ de solución enzimática de gabasa (GABA-T y SSDH, 2 unidades $/ \mathrm{ml}$ ) y 100 $\mu \mathrm{L}$ de sobrenadante de muestra y se agitó brevemente; hecho esto, se leyó la absorbancia inicial en un espectrofotómetro a $340 \mathrm{~nm}$, colocando el aparato en cero con una microcelda de referencia, la cual contenía todos los reactivos anotados anteriormente, pero en lugar de sobrenadante se le adicionaron $100 \mu \mathrm{L}$ de etanol al $75 \%$. Leída la absorbancia inicial, se agregaron $17 \mu \mathrm{L}$ de alfa-cetoglutarato $(0,02 \mathrm{M} ; \mathrm{pH}=7,9)$ en ambas microceldas y se incubaron a $25^{\circ} \mathrm{C}$ durante 30 minutos. Terminada la incubación, se leyó la absorbancia final de la muestra, colocando el aparato en cero con la microcelda de referencia. El aumento o disminución $(\Delta)$ en la absorbancia fue proporcional al contenido de GABA en las muestras $(8,9)$.

Este ensayo se basa en la siguiente reacción enzimática:

$\mathrm{GABA}+\alpha$-cetoglutarato $\underset{\text { semialdehído succínico + glutamato }}{\text { semialdehído succínico }+\mathrm{NADP}^{+} \longrightarrow}$
succinato $+\mathrm{NADPH}+\mathrm{H}^{+}$

Para la determinación de glutamato en las muestras, se pipetearon en una microcelda los siguientes reactivos: $980 \mu \mathrm{L}$ de tampón de glicinahidrazina $(0,5 \mathrm{M} / 0,4 \mathrm{M} ; \mathrm{pH}=9,0) ; 100 \mu \mathrm{L}$ de sobrenadante, $70 \mu \mathrm{L}$ de $\operatorname{NAD}^{+}(0,015 \mathrm{M})$; se leyó la absorbancia inicial en un espectrofotómetro a $340 \mathrm{~nm}$, colocando el aparato en cero con una microcelda de referencia que contenía, en lugar de sobrenadante, $100 \mu \mathrm{L}$ de etanol al $75 \%$. Leída la absorbancia inicial, se agregaron a ambas celdas $50 \mu \mathrm{L}$ de GDH (10 mg de proteína/ml) y se incubaron a $22^{\circ} \mathrm{C}$ durante 30 minutos.
Terminada la incubación, se leyó la absorbancia final de la muestra colocando en cero el aparato con la semi-microcelda de referencia. La variación en la absorbancia $(\Delta)$ fue proporcional al contenido de ácido glutámico en las muestras $(9,10)$.

Este ensayo se basa en la siguiente reacción enzimática:

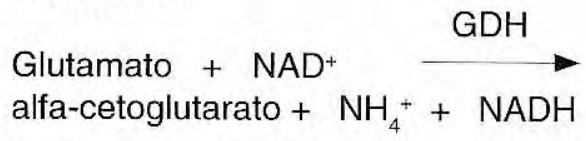

Para el ensayo de los niveles de aspartato se adicionaron, en su orden, en una microcelda espectrofotométrica: $1.084 \mu \mathrm{L}$ de solución tampón de fosfato $(0,066 \mathrm{M}, \mathrm{pH}=7,20), 200 \mu \mathrm{L}$ de sobrenadante, $20 \mathrm{~mL}$ de NADH $(0,012 \mathrm{M}), 8 \mu \mathrm{L}$ de GOT (2,5 mg de proteína/ml) y $8 \mu \mathrm{L} \mathrm{MDH}(2,5$ $\mathrm{mg}$ proteína/ml).

Esta mezcla se agitó manualmente y se leyó la absorbancia inicial a $340 \mathrm{~nm}$. Inmediatamente, para iniciar la reacción, se adicionaron $20 \mu \mathrm{L}$ de alfa-cetoglutarato $(0,1 \mathrm{M})$, se agitó e incubó a $37,5^{\circ} \mathrm{C}$ durante 10 minutos. Al término de la incubación, se leyó la absorbancia final. La variación entre la absorbancia final e inicial $(\Delta)$ fue directamente proporcional a la cantidad de aspartato contenido en los sobrenadantes $(9,11)$.

Este ensayo se basa en la siguientes reacciones enzimáticas:

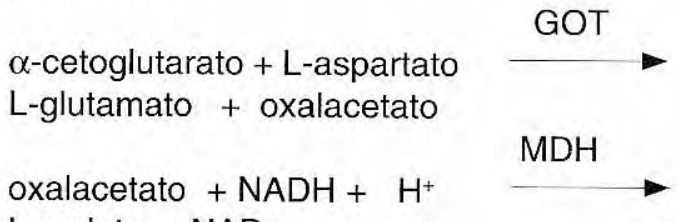

L-malato + NAD ${ }^{+}$

Las curvas de calibración de estos aminoácidos se elaboraron de acuerdo con los ensayos descritos arriba; se reemplazó el sobrenadante por las concentraciones respectivas de los estándares de GABA, glutamato y aspartato (en ácido perclórico $50 \mathrm{mM}$ ). Una vez obtenidas las $\Delta$ absorbancias, se utilizaron para elaborar las respectivas curvas de calibración. Para elaborar estas curvas, se adicionaron al ensayo concentraciones del aminoácido respectivo así: GABA 1, 3, 6 y 9 nanomoles; glutamato 1, 3, 5, y 7 nanomoles; aspartato $0,5,1,2$ y 3 nanomoles. 
Para obtener los valores de las muestras, en vez de los estándares, se adicionó un volumen correspondiente de sobrenadante, interpolando el valor de absorbancia a la respectiva curva para conocer el valor en nanomoles y, a partir de este dato, hacer los cálculos expresados en $\mu \mathrm{moles} / \mathrm{g}$ de tejido.

La determinación de GABA, glutamato y aspartato en cada sobrenadante se realizó por triplicado. La significación estadística de la diferencia entre los subgrupos basal, agudo y crónico de los estudios en el núcleo accumbens e hipocampo se estableció aplicando una prueba de ANOVA, de acuerdo con la prueba de Newman-Keuls (12). Todos los resultados se presentan como la media \pm el error estándar de la media. En todos los análisis se aceptó como estadísticamente significativo un nivel de confidencia del $95 \%$.

\section{Resultados}

El cuadro 1 y la figura 3 muestran el efecto de la administración aguda y crónica de cocaína sobre los niveles de los neurotransmisores estudiados en el núcleo accumbens de ratas; en todos los casos, la unidad de medida es $\mu$ moles/g de tejido fresco. Las medias \pm error estándar de las medias $(\bar{X} \pm E E M)$ de los niveles de GABA en los grupos control, agudo y crónico fueron de $3,61 \pm 0,094,3,80 \pm 0,089$ y $2,80 \pm 0,129$, respectivamente. Se observó una diferencia estadísticamente significativa en los niveles de GABA entre los grupos control y crónico $\left(q_{5,43}=7,66 ; p<0,01\right)$ y agudo y crónico $\left(q_{5,43}=11,39\right.$; $p<0,01)$.

Las medias \pm error estándar de los niveles de glutamato en los grupos control, agudo y crónico fueron de $10,42 \pm 0,178,14,83 \pm 0,168$ y $13,53 \pm$ 0,161 , respectivamente. Se observó una diferencia estadísticamente significativa en los niveles de glutamato entre los grupos control y agudo $\left(q_{5,43}=26,04 ; p<0,01\right)$ y control y crónico $\left(q_{5,43}=12,43 ; p<0,01\right)$.

Los niveles de aspartato en los grupos control, agudo y crónico (medias \pm error estándar) fueron de $2,25 \pm 0,064,3,28 \pm 0,026$ y $3,70 \pm 0,036$, respectivamente. Se observó una diferencia estadísticamente significativa en los niveles de
Cuadro 1. Efectos de la cocaína sobre los niveles de GABA, glutamato y aspartato en el núcleo accumbens de rata.

\begin{tabular}{lrrr}
\hline & \multicolumn{3}{c}{$(\overline{\mathrm{X}} \pm \mathrm{EE}) \mu \mathrm{mol} / \mathbf{g}^{\cdot}$} \\
\cline { 2 - 4 } & \multicolumn{1}{c}{ Control } & \multicolumn{1}{c}{ Agudo } & \multicolumn{1}{c}{ Crónico } \\
\hline Gaba & $3,61 \pm 0,094$ & $3,80 \pm 0,089$ & $2,80 \pm 0,129$ \\
Glutamato & $10,42 \pm 0,178$ & $14,83 \pm 0,168$ & $13,53 \pm 0,161$ \\
Aspartato & $2,25 \pm 0,064$ & $3,28 \pm 0,026$ & $3,70 \pm 0,036$ \\
\hline
\end{tabular}

*Cada valor es el promedio y el error estándar de diez animales; ensayos por triplicado.

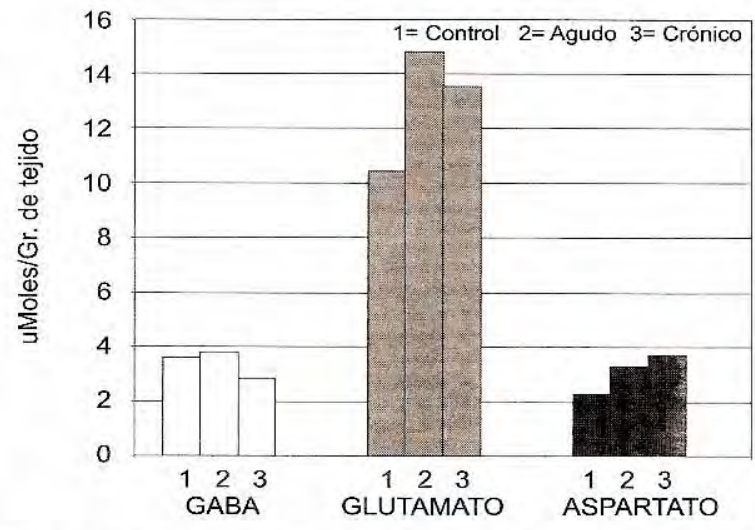

Figura 3. Efectos de la cocaína sobre los niveles de GABA, glutamato y aspartato en el núcleo accumbens de ratas. Diferencias significativas de GABA: entre control/crónico y agudo/crónico; glutamato y aspartato: entre control/agudo y entre control/crónico.

aspartato entre los grupos control y agudo $\left(q_{5,43}=22,82 ; p<0,01\right)$ y control y crónico $\left(q_{5,43}=30,92 ; p<0,01\right)$.

El cuadro 2 y la figura 4 muestran el efecto de la administración aguda y crónica de cocaína sobre los niveles de GABA, glutamato y aspartato en el hipocampo de ratas; en todos los casos la unidad de medida es $\mu \mathrm{moles} / \mathrm{g}$ de tejido fresco. Las medias \pm error estándar de las medias de los niveles de GABA en los grupos control, agudo y crónico fueron de $4,42 \pm 0,085,4,62 \pm 0,131$ y $3,42 \pm 0,110$, respectivamente. Se observó una diferencia estadísticamente significativa en los niveles de GABA entre los grupos control y crónico $\left(q_{5,43}=6,31 ; p<0,01\right)$ y agudo y crónico $\left(q_{5,43}=8,11\right.$; $p<0,01)$.

Las medias \pm error estándar de los niveles de glutamato en los grupos control, agudo y crónico fueron de $12,39 \pm 0,155,18,19 \pm 0,160$ y $17,41 \pm$ 
Cuadro 2. Efectos de la cocaína sobre los niveles de GABA, glutamato y aspartato en el hipocampo de rata.

\begin{tabular}{lrrr}
\hline & \multicolumn{3}{c}{$(\overline{\mathrm{X}} \pm \mathrm{EE}) \boldsymbol{\mu \mathrm { mol } / \mathbf { g } ^ { * }}$} \\
\cline { 2 - 4 } & \multicolumn{1}{c}{ Control } & \multicolumn{1}{c}{ Agudo } & \multicolumn{1}{c}{ Crónico } \\
\hline Gaba & $4,42 \pm 0,085$ & $4,62 \pm 0,131$ & $3,42 \pm 0,110$ \\
Glutamato & $12,39 \pm 0,155$ & $18,19 \pm 0,160$ & $17,41 \pm 0,137$ \\
Aspartato & $2,58 \pm 0,071$ & $3,59 \pm 0,068$ & $3,85 \pm 0,033$ \\
\hline
\end{tabular}

*Cada valor es el promedio y el error estándar de diez animales; ensayos por triplicado.

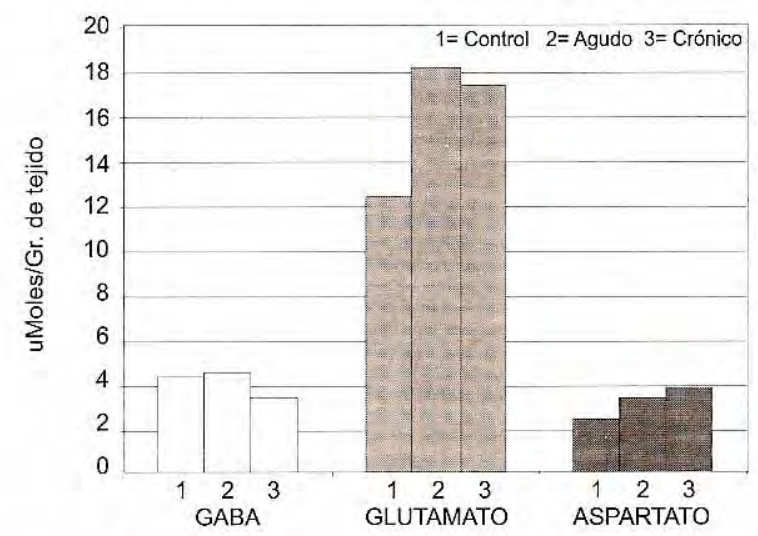

Figura 4. Efectos de la cocaína sobre los niveles de GABA, glutamato y aspartato en el hipocampo de rata. Diferencias significativas de GABA entre control/crónico y agudo/crónico y de glutamato y aspartato entre control/agudo y control/ crónico.

0,137 , respectivamente. Se observó una diferencia estadísticamente significativa en los niveles de glutamato entre los grupos control y agudo $\left(q_{5,43}=38,32 ; p<0,01\right)$ y control y crónico $\left(q_{5,43}=26,57 ; p<0,01\right)$.

Las medias \pm error estándar de los niveles de aspartato en los grupos control, agudo y crónico fueron de $2,58 \pm 0,071,3,59 \pm 0,068$ y $3,85 \pm 0,033$, respectivamente. Se observó una diferencia estadísticamente significativa en los niveles de aspartato entre los grupos control y agudo $\left(q_{5,43}=13,40 ; p<0,01\right)$ y control y crónico $\left(q_{5,43}=21,01 ; p<0,01\right)$.

\section{Discusión}

Con relación a los interrogantes clínicos y básicos de los fenómenos, procesos y mecanismos íntimos de la dependencia y abuso de la cocaína, observamos un gran número de investigaciones en las últimas décadas que tratan de ahondar en el conocimiento y explicaciones de esta conducta. Se ha profundizado mucho, especialmente en los aspectos experimentales básicos de este fenómeno, del papel de núcleos, vías, receptores y neurotransmisores. Incluso la investigación génica de receptores involucrados se está ampliando $(3,5,6,13)$.

Los resultados muestran la gran relevancia de la actividad de diferentes sustancias como la dopamina, el GABA, el glutamato, el aspartato y algunos neuropéptidos en la génesis y permanencia de la adicción, no sólo de cocaína, sino también de otras sustancias de abuso.

Una extensa evidencia sugiere una hipótesis dopaminérgica para las propiedades reforzadoras de la cocaína. De acuerdo con ésta, el principal mecanismo de la cocaína es la unión a los transportadores presinápticos para la dopamina, inhibiendo principalmente la recaptación de este neurotransmisor, lo que resulta en la potenciación de la transmisión dopaminérgica en las vías mesocorticolímbicas $(3,14)$.

Este modelo ha servido para la utilización de fármacos dopaminérgicos en el tratamiento del abuso y dependencia a la cocaína, tanto en modelos experimentales con roedores como en estudios clínicos en humanos. Sin embargo, este enfoque terapéutico no ha proporcionado mejoría o cura en la totalidad de los casos, lo cual sugiere que otros sistemas podrían tener papel importante en este fenómeno (15).

Precisamente, las observaciones de varios autores sobre los sistemas GABAérgicos, glutamatérgicos, monoaminérgicos y peptidérgicos así lo sugieren. Se ha postulado que la administración de cocaína disminuiría la actividad GABAérgica e incrementaría la actividad glutamatérgica y peptidérgica opioide. Además, se ha observado que los antagonistas de NMDA bloquean la excitación producida por la cocaína y las anfetaminas, lo cual está a favor del papel relevante del sistema glutamatérgico $\mathrm{y} / \mathrm{o}$ aspartatérgico en los efectos agudos de estas sustancias de abuso que producen adicción $(16,17)$. 
Estudios in vitro han mostrado que el ácido glutámico favorece la liberación de la dopamina en el núcleo accumbens. En este sentido, se ha observado, tanto in vitro como in vivo, que el glutamato o agonistas glutamatérgicos inducen la liberación de dopamina. Esta regulación positiva recíproca tendría una gran relevancia en la fisiología y farmacología de la actividad sicomotriz relacionada con este núcleo (17).

La cocaína produce alteración de la actividad GABAérgica en el núcleo accumbens. Se ha informado la disminución de la población de receptores GABA y un incremento en el recambio sináptico de GABA en el estriado. En el hipocampo, los estudios electrofisiológicos muestran, además, disminución de la actividad inhibitoria después de la administración de cocaína $(18,19)$.

Con la administración crónica de cocaína se da de manera moderada una disminución (alrededor del $23 \%$ ) de los niveles de GABA tanto en el núcleo accumbens como en el hipocampo. La estimu-lación crónica de proyecciones dopaminérgicas del mesencéfalo ventral hacia el núcleo accumbens disminuiría los valores del GABA, lo que se explica por la interrelación inhibitoria recíproca entre dopamina y GABA. Estos resultados podrían sugerir, además, una acción directa inhibitoria de las neuronas GABAérgicas por cocaína. También otros sistemas como el serotoninérgico podrían ser afectados por la cocaína y éste, a su vez, modular el sistema GABAérgico $(20,21)$.

La administración aguda de cocaína produce en forma muy significativa un incremento del glutamato y del aspartato, tanto en el núcleo accumbens como en el hipocampo (alrededor del $40 \%$ ). Este aumento concuerda con observaciones que muestran que las actividades glutamatérgica y aspartatérgica se incrementan con la administración aguda de cocaína (17).

La administración crónica de cocaína produjo un aumento similar de glutamato y aspartato, tanto en el núcleo accumbens como en el hipocampo. La transmisión glutamatérgica/aspartatérgica es modulada por receptores dopaminérgicos y puede producir incremento de sus niveles directamente.
Este sería el mecanismo que utilizaría la cocaína para incrementar estas actividades. De acuerdo con estos resultados, se ha demostrado que la administración de antagonistas de receptores NMDA puede bloquear o antagonizar los efectos motores, estimulantes, neurotóxicos y reforzadores de la conducta o la administración de la cocaína (22-24).

Toda la información y los resultados anteriores apoyarían la hipótesis de que las actividades del GABA, del glutamato y del aspartato en sus núcleos, terminales y receptores son de relevancia en las acciones y efectos agudos y crónicos de la cocaína en el SNC de mamíferos, lo cual estaría favorecido por la interregulación positiva entre la actividad dopaminérgica y el glutamato/aspartato; la actividad dopaminérgica disparada (no necesariamente a expensas del aumento de los niveles de dopamina) por la administración crónica de cocaína produciría liberación de la actividad de estos dos neurotransmisores excitadores. La disminución observada de la actividad GABAérgica favorecería aún más la hipersensibilidad de la actividad excitadora en la condición crónica.

Esta hipótesis aumenta las posibilidades farmacoterapéuticas en el tratamiento de la dependencia y la adicción a sustancias de abuso. Por tanto, la experimentación para el uso de GABA agonistas o bloqueantes de receptores glutamatérgicos es una nueva perspectiva.

\section{Agradecimientos}

Nuestro agradecimiento a la Universidad de Cartagena y a Colciencias por el apoyo para la realización de esta investigación.

\section{Referencias}

1. Lorenzo P, Leza JC, Lizasoain I. Drogodependencias. En: Velasco A, Lorenzo P, Serrano JS, Andres-Trelles F, editores. Velázquez farmacología. $16^{a}$ ed. Madrid: Mcgraw-Hill-Interamericana de España; 1993. p.498.

2. Escohotado A. Las drogas. De los orígenes a la prohibición. Madrid: Alianza Editorial; 1994. p.5-11.

3. Ulloque RA. Sistema cerebral del placer y de la drogodependencia. Biomédica 1999;19:321-30.

4. Stein L. Self-stimulation of the brain and the central stimulant of amphetamine. Fed Proc 1964;23:836-41. 
5. Tassin JP. Drogas, dependencia y dopamina. Mundo Científico 1998;189:68-73.

6. Bardo MT. Neuropharmacological mechanism of drug reward: beyond dopamine in the nucleus accumbens. Crit Neurobiol 1998;12:37-67.

7. Paxinos G, Watson C. The rat brain in stereotaxic coordinates. 2nd. ed. San Diego: Academic Press; 1986.

8. Fahn S, Cote LJ. Regional distribution of GABA in brain of the rhesus monkey. J Neurochem 1968;15:209-15.

9. Graham LT, Aprison MH. Distribution of some enzymes associated with metabolism of glutamate, aspartate, GABA and glutamine in cat spinal cord. J Neurochem 1969;16:559-66.

10. Bernt E, Bergmeyer HU. L-glutamate. Determination with glutamic dehydrogenase. En: Bermeyer $\mathrm{HU}$, editor. Methods of enzymatic analysis. New York: Academic Press; 1963. p.384-9.

11. Pfleiderer G. L-aspartic. Determination with glutamateoxalacetate transaminase and malic dehydrogenase. En: Bermeyer $\mathrm{HU}$, editor. Methods of enzymatic analysis. New York: Academic Press; 1963. p.381-3.

12. Tallarida RJ, Murria RB. Newman-Keuls test. En: Manual of pharmacologic calculations with computer programs. 2nd. edición: New York: Springer-Verlag; 1987. p.121-5.

13. Graybel AM. Acute effects of psychomotor stimulant drugs on gene expression in the striatum. En: Grzamma $\mathrm{R}$, Brown RM, editors. Activation of inmediate early genes by drugs of abuse. National Institute of Drug Abuse Research Monography Series. Washington, D.C.: U.S. Government Printing Office; 1993. p.125:72-81.

14. Ulloque RA. Farmacología de la cocaína. Tribuna Médica 1999;99:219-28.
15. Pastrana A, Ulloque RA. Cocainodependencia: neurobioquímica y fármaco-terapéutica. Tribuna Médica 1996;94:308-16.

16. Kelley AE, Thorne LC. NMDA receptors mediated the behavioral effects of amphetamine infused into the nucleus accumbens. Brain Res Bull 1992;29:244-54.

17. Moghaddam B, Gruen RJ, Roth RH, Bunney BS, Adams RN. Effects of alfa-glutamate on the release of striatal dopamine. In vivo dialysis and electrochemical studies. Brain Res 1990;518:55-60.

18. Guerrero RF, Guerrero AR. Cambios de excitabilidad neuronal hipocampal durante la administración de cocaína. Acta Médica de Cartagena 1991;3:23-30.

19. Kushner SA, Dewey SL, Kornetsky C. Gamma-vinyl GABA attenuates cocaine-induced lowering of brain stimulation reward thresholds. Psychopharmacology (Berlin) 1997;133:383-88.

20. Hieden JAM, Van DER, Venema K, Korf J. In vivo release of endogenous GABA from rat striatum: inhibition by dopamine. J Neurochem 1980;34:1338-41.

21. Camerun DL, Williams JT. Cocaine inhibits GABA release in the vta through endogenous $5 \mathrm{HT}$. J Neurosci 1994;14:6763-7.

22. Kalivas PW, Duffy P. $D_{1}$ receptor modulates glutamate transmission in the vta. J Neurosci 1995;15:5379-88.

23. Smith JA, Mo Q, Guo H, Kunko PM, Robinson SE. Cocaine increases extraneuronal levels of aspartate and glutamate in the nucleus accumbens. Brain Res 1995; 683:264-9.

24. McGinty JF. Introduction to the role of excitatory aminoacids in the physiology of abused drugs. Drug Alcohol Depend 1995;37:91-4. 